colleagues examined whether cessation of treatment before surgery affects postoperative complication rates.

This retrospective, single-center study identified 92 patients who had undergone a total of 127 surgical procedures during a course of treatment with at least one TNF blocker (etanercept, infliximab or adalimumab) between 1997 and 2004 . The majority $(77.2 \%)$ of patients had rheumatoid arthritis, $20 \%$ had spondylarthropathies, and the remainder had other rheumatic diseases. The most common procedures were orthopedic operations (84.3\%), such as joint replacements (31.5\%) and arthrodeses (24.4\%).

Postoperative complications (such as infection, thrombosis and healing complications occurring within 1 month post-surgery) were observed after 24 (18.9\%) of 127 operations. The overall rate of postoperative complications that could potentially be related to TNF inhibitor use was $15 \%$. Postoperative complication rates did not differ significantly between patients who discontinued anti-TNF treatment $>5$ half-lives before surgery and those who either discontinued nearer to surgery or did not stop treatment at all.

The authors conclude that the postoperative complication rates observed in this study are higher than in previous reports; however, they advise that patients using TNF inhibitors should be carefully monitored for postoperative complications until further studies examining this association are performed.

Original article Ruyssen-Witrand A et al. (2007) Complication rates of 127 surgical procedures performed in rheumatic patients receiving tumor necrosis factor alpha blockers. Clin Exp Rheumatol 25: 430-436

\section{Intrathecal morphine infusion therapy improves chronic pain in patients with osteoporosis}

Vertebral fracture is common in patients with osteoporosis, and has a marked effect on quality of life (QOL). Chronic pain can occur in patients who have experienced multiple fractures; however, treating the pain with systemic analgesics can cause adverse effects in some patients, and can also cause undesirable interactions with drugs used to treat other comorbid illnesses. Shaladi and colleagues report their experience of using intrathecal morphine infusion, delivered by an implanted pump, to treat chronic pain refractory to conventional opioid delivery.

The authors treated 24 patients (mean age 74.3 years, 19 women) with advanced osteoporosis who had scores $>7$ on a pain visual analog scale (VAS) after 3 months of noninvasive therapies and 1 month of oral or transdermal opioid use. QOL was measured using the Quality of Life Questionnaire of the European Foundation of Osteoporosis (QUALEFFO). The pain VAS and the QOL questionnaire were administered at baseline, implantation and after 1 year's follow-up. The mean morphine dose at implantation was $0.33 \mathrm{mg} / \mathrm{h}$, increasing to $0.68 \mathrm{mg} / \mathrm{h}$ after 1 year's follow-up.

Significant improvements were observed in all pain and QOL measures for all patients at 1 year follow-up, including the pain VAS (mean 8.71 at baseline, versus 1.92 at 1 year followup), and all QUALEFFO categories, such as ambulation. After implantation, adverse effects were limited to nausea $(n=3)$, wound infection $(n=1)$ and delayed healing $(n=1)$; no patients required supplementary analgesics.

The authors conclude that the pain relief, QOL improvements and low incidence of adverse effects observed in this study support the use of intrathecal therapy when other conventional treatments are unsuccessful.

Original article Shaladi A et al. (2007) Continuous intrathecal morphine infusion in patients with vertebral fractures due to osteoporosis. Clin J Pain 23: 511-517

\section{Autoantibodies can be detected before onset of SLE symptoms}

The many possible clinical presentations of systemic lupus erythematosus (SLE) can make diagnosis difficult. Heinlen et al. conducted a retrospective review of the military hospital records of 130 patients with SLE to identify patterns characteristic of early clinical events in SLE and to establish whether SLE-associated autoantibodies can be detected before the development of clinical symptoms.

Of the 130 patients, 104 met at least one of the American College of Rheumatology (ACR) criteria for SLE before diagnosis; the remaining patients had sudden-onset SLE. Discoid rash and seizures had the earliest onset, appearing an average of 1.74 and 1.70 years, respectively, before diagnosis of SLE. Arthritis was the most common symptom, being present 